\title{
Neuro-Fuzzy Sensor Fault Diagnosis of an Induction Motor
}

\author{
M. L. Benloucif
}

Automatic Control Laboratory, LAS, Department of Electrical Engineering,

Faculty of Engineering, University of Skikda, Algeria

Received 20 January 2010; accepted 2 March 2010

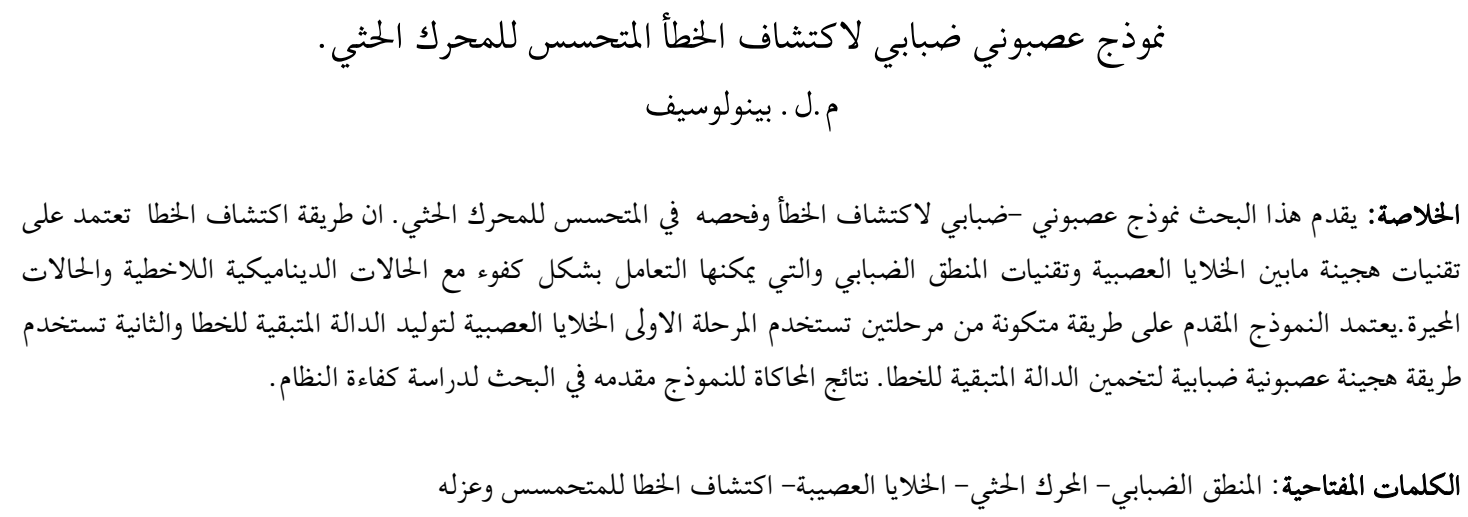

Abstract: In this paper, a neuro-fuzzy fault diagnosis scheme is presented and its ability to detect and isolate sensor faults in an induction motor is assessed. This fault detection and isolation (FDI) approach relies on a combination of neural modelling and fuzzy logic techniques which can deal effectively with nonlinear dynamics and uncertainties. It is based on a two step neural network procedure: a first neural network is used for residual generation and a second fuzzy neural network performs residual evaluation. Simulation results are given to demonstrate the efficiency of this FDI approach.

Keywords: Fuzzy logic, Induction motor, Neural networks, Sensor fault detection and isolation

\section{Introduction}

The problem of fault detection and isolation (FDI) is a crucial issue for the safety, reliability and performance of industrial processes.

The usual approach to fault diagnosis is based on hardware redundancy (multiple sensors, actuators and components) and uses a voting technique to decide if a fault has occurred and to locate it among the redundant system elements (Frank 1990). Instead, the analytical redundancy FDI approach, also referred to as the model-based FDI approach, makes use of a mathematical model of the monitored system [(Frank 1990). The task of model based diagnosis methods consists of detecting faults that may occur in the system and which can be additive or multiplicative in nature.

Basically the FDI procedure consists of two main steps: generation of residuals which should be useful

\footnotetext{
*Corresponding author's e-mail: m.1.benloucif@gmail.com
}

fault indicators, and residual evaluation which involves decision making. The model-based FDI approach which has received intensive attention uses mainly state and parameter estimation techniques (Frank 1990). Model based FDI performance is directly related to the accuracy of the mathematical model of the monitored system. The effect of model uncertainties, disturbances and noise is therefore a key issue in model based fault diagnosis.

The main design requirements of model based fault diagnosis procedures are thus concerned with the problems of robustness with respect to model uncertainties and enhancement of sensitivity to faults. These requirements are contradictory so a trade off is needed to cope with sources of false alarms and missed detections. Two strategies may be used: an active strategy consisting in robust residual generation and a passive one through robust residual evaluation. Most of the 
existing model based FDI techniques rely on the use of linear system models (Benloucif and Staroswiecki 2002 and Frank 1990). Often, nonlinear systems are described by linear models with additive disturbances. Robust residual generation based on unknown input observers to achieve disturbance decoupling may provide an efficient solution to fault detection and isolation problems. As far as linear systems are concerned the problem of robust residual generation may be considered to be mature ((Benloucif and Staroswiecki 2002, Frank 1990 and Patton and Chen 1997) whereas the FDI problem for nonlinear dynamic systems has been investigated to a lesser extent (Benloucif and Balaska 2006; Garcia and Frank 1997 and Jiang et al. 2001).

Alternatively, FDI can be performed using qualitative techniques such as expert systems, fuzzy logic, neural networks (Al; exandru et al. 2000; Benloucif and Mehennaoui 2002; Benloucif and Mehennaoui 2005; Chen and Lee 2002; Evsukoff et al. 1999; Frank 1990; Isermann 1998; Schneider and Frank 1996; Simani and Fantuzzi 2002; Takagi and Sugeno 1985, Theilliol et al. 1997 and Uppal et al. 2002). To overcome the limitations of the analytical FDI approach, the actual trend integrates model based (analytical) and knowledge based (non analytical) methods in order to take advantage of their respective performances. Residual generation and residual evaluation for decision making may be achieved by using appropriate combinations of different techniques such as state estimation, parameter estimation, neural networks, fuzzy logic inference.

In (Benloucif and Mehennaoui 2002) a fault diagnosis procedure for linear systems used a combination of an analytical residual generator based on Kalman filtering and a fuzzy neural network for residual evaluation. In this work, an extension of the neuro-fuzzy FDI scheme given in (Benloucif and Balaska 2006) is proposed. It is based on a two step neural network procedure: The first network which has the ability to model a wide class of nonlinear dynamic systems acts as an on-line residual generator. The second network performs the decision making which consists in detecting and isolating a fault when it occurs. This neural network coupled to a fuzzy inference block acts as an on-line fault classifier.

The paper is organized as follows. In section 2 the model of the induction motor is presented, starting from the classical Park transformation. The architecture of the neuro-fuzzy scheme used for residual generation and evaluation is discussed in section 3 . Simulation results are given in section 4 to illustrate the performance of the proposed neuro-fuzzy FDI scheme for sensor fault diagnosis of the induction motor.

\section{Model of the Induction Motor}

Assuming linear magnetic circuits and a balanced three-phase system in the $(a, b, c)$ frame, the electrical equations of the induction motor expressed in the two-phase stationary $(d, q)$ reference frame (Benloucif and Balaska 2006) are:

$$
\begin{aligned}
& {\left[\begin{array}{c}
\Phi_{s d} \\
\Phi_{s q} \\
\Phi_{r d} \\
\Phi_{r q}
\end{array}\right]=\left[\begin{array}{cccc}
L_{s} & 0 & L_{m} & 0 \\
0 & L_{s} & 0 & L_{m} \\
L_{m} & 0 & L_{r} & 0 \\
0 & L_{m} & 0 & L_{r}
\end{array}\right]\left[\begin{array}{c}
I_{s d} \\
I_{s q} \\
I_{r d} \\
I_{r q}
\end{array}\right]} \\
& V_{s d}=R_{s} I_{s d}+\dot{\Phi}_{s d}-\Phi_{s q} \dot{\theta}_{s} \\
& V_{s q}=R_{s} I_{s q}+\dot{\Phi}_{s q}+\Phi_{s d} \dot{\theta}_{s} \\
& V_{r d}=0=R_{r} I_{r d}+\dot{\Phi}_{r d}-\Phi_{r q} \dot{\theta}_{r} \\
& V_{r q}=0=R_{r} I_{r q}+\dot{\Phi}_{r q}+\Phi_{r d} \dot{\theta}_{r}
\end{aligned}
$$

where $\Phi, I, V$ are the stator/rotor fluxes, currents and voltages expressed in the $(d, q)$ reference frame. $\theta_{s}$ is the angle between the stator reference frames $(a, b, c)$ and $(d, q)$, and $\theta_{r}$ is the angle between the rotor reference frames $(a, b, c)$ and $(d, q) . R_{s}, R_{r}, L_{s}, L_{r}$ are the stator/rotor resistances and inductances, respectively, and $L_{m}$ is the magnetizing inductance. For a squirrelcage IM the rotor voltages are zero. The mechanical equation is:

$$
J \dot{\Omega}=T_{e}-f_{\mathcal{V}} \Omega-T_{L}
$$

and dthe electromagnetic torque $T_{e}$ is given by:

$$
T_{e}=p L_{m}\left(I_{s q} I_{r d}-I_{s d} I_{r q}\right)
$$

$\Omega, J, f_{v}$ are the rotor speed, inertia and friction, respectively, $p$ is the number of pole pairs and $T_{L}$ is the load torque. Using the Park transformation with the reference frame $(d, q)$ fixed to the stator (ie with $\theta_{s}=0$ and $\dot{\theta}_{r}=-p \Omega$ ), Eq. (1),(2),(3) and (4) are transformed to the following nonlinear state space equations: 


$$
\begin{aligned}
\dot{x} & =\left[\begin{array}{l}
\xi\left(-L_{r} R_{S} x_{1}+L_{m}^{2} p x_{5} x_{2}+L_{m} R_{r} x_{3}+p L_{r} L_{m} x_{5} x_{4}\right) \\
\xi\left(-L_{m}^{2} p x_{5} x_{1}-L_{r} R_{S} x_{2}-p L_{r} L_{m} x_{5} x_{3}+L_{m} R_{r} x_{4}\right) \\
\xi\left(L_{m} R_{S} x_{1}-p L_{s} L_{m} x_{5} x_{2}-L_{S} R_{r} x_{3}-L_{S} L_{r} p x_{5} x_{4}\right) \\
\xi\left(p L_{s} L_{m} x_{5} x_{1}+L_{m} R_{S} x_{2}+L_{S} L_{r} p x_{5} x_{3}-L_{S} R_{r} x_{4}\right) \\
\frac{1}{J}\left(p L_{m} x_{2} x_{3}-p L_{m} x_{1} x_{4}-f_{v} x_{5}-T_{L}\right)
\end{array}\right] \\
& +\left[\begin{array}{lr}
\xi L_{r} & 0 \\
0 & \xi L_{r} \\
-\xi L_{m} & 0 \\
0 & -\xi L_{m} \\
0 & 0
\end{array}\right] u \quad, \quad y=\left[\begin{array}{lllll}
1 & 0 & 0 & 0 & 0 \\
0 & 1 & 0 & 0 & 0 \\
0 & 0 & 0 & 0 & 1
\end{array}\right] x
\end{aligned}
$$

Where the state, input and output vectors are defined as:

$$
\begin{aligned}
& x=\left(\begin{array}{lllll}
I_{s d} & I_{s q} & I_{n d} & I_{r q} & \Omega
\end{array}\right)^{T}, u=\left(\begin{array}{ll}
V_{s d} & V_{s q}
\end{array}\right)^{T} \\
& y=\left(\begin{array}{lll}
I_{s d} & I_{s q} & \Omega
\end{array}\right)^{T} \quad \text { and } \xi=1 /\left(L_{r} L_{s}-L_{m}^{2}\right)
\end{aligned}
$$

The stator currents $I_{s d}, I_{s q}$ and the rotor speed $\Omega$ are assumed to be measured since they are usually used for control purposes. As a matter of fact, measurements of stator currents and voltages are made in the $(a, b, c)$ reference frame but they can be expressed in the $(d, q)$ reference frame and viceversa, thanks to the Park transformation:

$$
\left[\begin{array}{l}
V_{s d} \\
V_{s q}
\end{array}\right]=\sqrt{\frac{2}{3}}\left[\begin{array}{rrr}
1 & -\frac{1}{2} & -\frac{1}{2} \\
0 & \frac{\sqrt{3}}{2} & -\frac{\sqrt{3}}{2}
\end{array}\right]\left[\begin{array}{l}
V_{a} \\
V_{b} \\
V_{c}
\end{array}\right]
$$

In the next sections we present a particular neurofuzzy FDI procedure and its application for sensor fault diagnosis of an induction motor.

\section{Neuro-Fuzzy Based Residual Genera- tion and Evaluation}

Neuro-fuzzy FDI systems can be designed as hybrid systems in which neural networks and fuzzy system modules may cooperate and interact to implement efficiently the required FDI tasks. Indeed, fuzzy logic systems can be combined with neural networks to design neuro-fuzzy structures whose successful applications rely on the ease of rule base design, linguistic modelling, applicability to complex uncertain and nonlinear systems, learning abilities, parallel processing.

A general FDI structure which uses any combination of neural, fuzzy and neuro-fuzzy methods for residual generation and evaluation is depicted in Fig. 1.

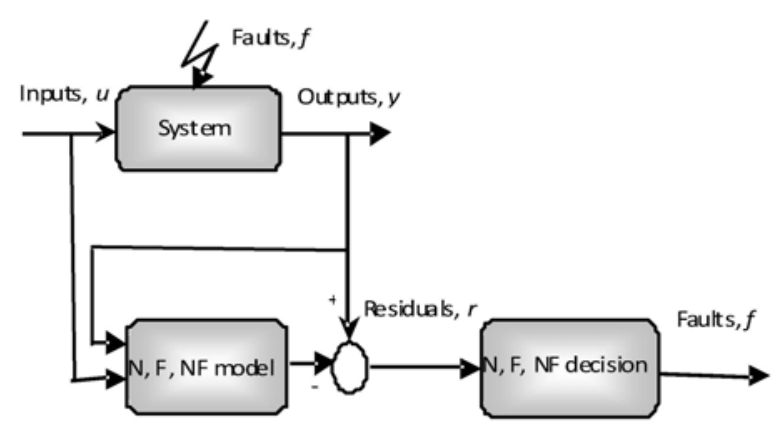

Figure 1. Neuro fuzzy FDI structure

\subsection{Residual Generation}

It is relevant to use the high potential of neural networks for nonlinear system modelling in the context of fault diagnosis of nonlinear dynamic systems. The neural network architecture most commonly used is the multilayer perceptron (MLP) network (Norgaard et al. 2000). The configuration for neural model identification is depicted in Fig. 2. Its implementation goes through the following steps.

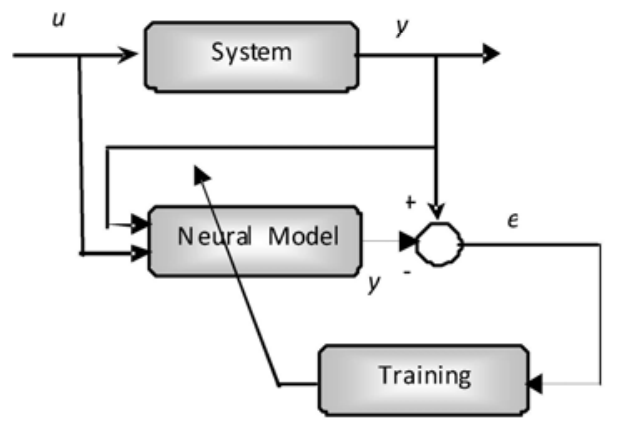

Figure 2. Neural network identification

\subsubsection{Off-Line Construction of a Database}

Expert knowledge of the process characteristics is used under different operating conditions.

\subsubsection{Selection of the Neural Network Structure}

Assuming that an input-output data set $\{u(k), y(k)\}$ $k=I, \ldots ., N$ has been obtained, a nonlinear model structure is chosen in the general predictor form given by Eq. (7).

$$
\hat{y}(k)=g(\varphi(k), \theta)
$$

where the regression vector $\varphi(k)$ has to be selected and is the vector of adjustable parameters known as the weights of the network and $g$ is the nonlinear function which must be realized by a suitable neural network architecture.

The NNARMAX model is obtained with the following regression vector definition, where $\varepsilon(k)=y(k)-\hat{y}(k):$ 
$\varphi^{T}(k)=(y(k-1) \ldots y(k-n), u(k-d) \ldots u(k-d-m) \varepsilon(\mathrm{k}-1) \ldots \varepsilon(k-$ n'))

The choice of this regressor form leads to a predictor with feedback which can be implemented as a recurrent neural network.

The NNARX model is recommended (Chen and Lee, 2002 and Norgfaard et al. 2000) when the system under consideration is deterministic or weakly noisy and this is the case for our application on an induction motor. The NNARX model is defined by the regression vector: $\varphi^{T}(k)=(y(k-1) \ldots y(k-n), u(k-d) \ldots u(k-d-m)$.

A multivariable NNARX model can be adequately implemented as a feedforward two-layer perceptron network having one hidden layer and an output layer as shown in Fig. 3.
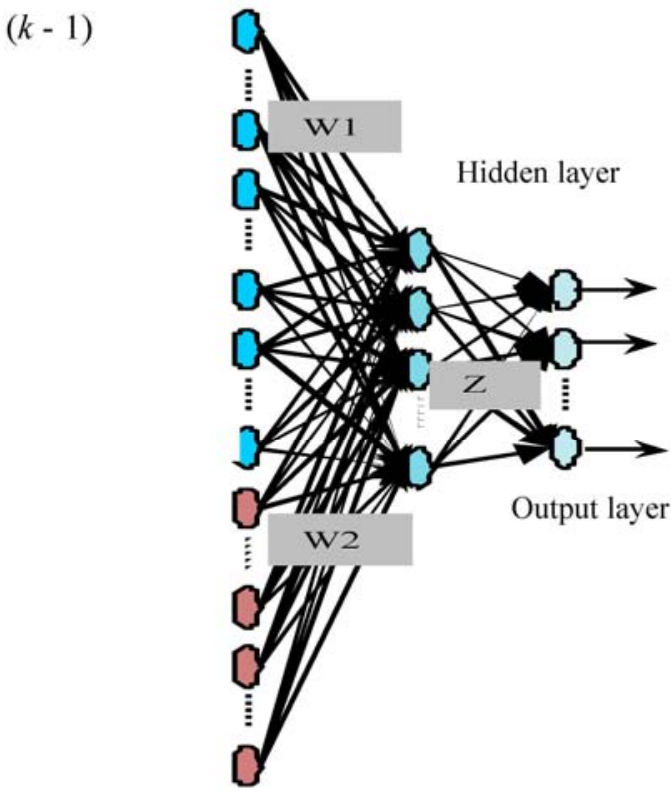

Figure 3. Two-layer neural network

The vector $\varphi(k)$ of delayed outputs and inputs of the system is applied to the network inputs. $\left(n_{1} \ldots n_{n}\right.$, $\left.m_{1} \ldots m_{m}, d\right)$ are the structural indices also referred to as the lag space of the neural model. The input delay $d$ is generally taken as one. The hidden layer includes a sufficient number $n_{h}$ of sigmoid units ( $n_{h}$ must be specified experimentally) and the output layer contains linear units.

$W=\left(\begin{array}{ll}W 1 & W 2\end{array}\right)=\left(W_{i j}\right)$ is the weight matrix relating the inputs to the hidden layer units and $Z=\left(Z_{i f}\right)$ is the weight matrix relating the hidden layer units to the output units.

The neural network outputs are given by:

$$
\begin{aligned}
& \hat{y}_{i}(k)=\psi_{i}\left(\sum_{j=1}^{n_{h}} Z_{i j} h_{j}(k)+z_{i 0}\right) \quad i=1 . . . n \\
& h_{j}(k)=\phi_{j}\left(\sum_{l=1}^{n_{\varphi}} W_{j l} \varphi_{l}(k)+w_{j 0}\right) \quad j=1 . . n_{h}
\end{aligned}
$$

where $\varphi_{j}$ are sigmoïd type activation functions and $\Psi_{i}$ are linear type activation functions and $\left(w_{j o}, z_{i o}\right)$ are the biases.

\subsubsection{Network Training}

The network weights and biases (randomly initialized) are adjusted using a suitable minimisation algorithm of the following mean square error criterion:

$$
E_{N}=\frac{1}{N} \sum_{k=1}^{N}(y(k)-\hat{y}(k))^{T}(y(k)-\hat{y}(k))
$$

where $N$ is the length of the training data set. The Levenberg-Marquardt algorithm is recommended to use as pointed out in (Norgaard et al. 2000).

\subsection{Network Validation}

In this stage the resulting neural model is evaluated to decide for its adequate representation of the system. This is done by testing the trained network using a data set different from the one used for training. If the trained network is judged unsatisfactory after the validation tests then it is necessary to go backwards in the procedure by retraining the network with different weight initializations, or by generating additional training data, or by modifying the network structure (by redefining the regression vector and the number of hidden units).

All these steps are accomplished off-line. When the neural network is validated, it may be utilized for online residual generation.

\subsubsection{Residual Evaluation}

The task of residual evaluation can be achieved by a neuro-fuzzy decision scheme as represented in Fig. 4.

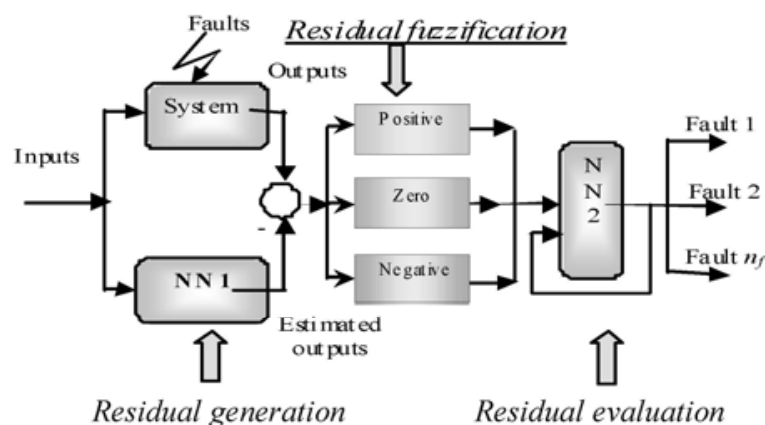

Figure 4. Fuzzy neural diagnostic scheme 
A neuro-fuzzy network is based on the association of fuzzy logic inference and the learning ability of neural networks.

The neuro-fuzzy approach is a powerful tool for solving important problems encountered in the design of fuzzy systems such as: determining and learning membership functions, determining fuzzy rules, adapting to the system environment.

The main points of the residual evaluation procedure are described below.

\subsubsection{Residual Fuzzification}

It consists in converting the numerical values of residuals into linguistic variables. Each input (residual) may be described by three linguistic variables (Negative, Zero, Positive). Each linguistic variable is represented by a membership function which has generally a triangular or trapezoidal shape. The linguistic variable Zero defines the range where the residual may be considered to be unaffected by a fault. The linguistic variables Negative and Positive define the residual amplitude ranges indicating the presence of a fault. The corresponding membership functions give the extent to which a residual is or is not affected by a fault.

\subsubsection{Neural Network Structure}

For fault diagnosis it is desirable to use a neural network to model the nonlinear relationship between the fuzzified residuals and the fault decision functions. A multilayer perceptron network is therefore a good candidate. Moreover, to account for memory in the decision process it is necessary to use a recurrent neural network (RNN). The RNN may be implemented as a neural model described by:

$$
D_{k}\left(f_{i}\right)=g_{i}(\varphi(k))
$$

where $D_{k}\left(f_{i}\right), i=1 \ldots n_{f}$, are the fault decision functions also referred to as fault indicators and $f_{i}$ are the faults acting on the process. The regression vector contains the fuzzy residuals $R_{i}(k), \quad i=1 \ldots n_{r}$, and the delayed decisions $D_{k-1}\left(f_{i}\right), \quad i=1 \ldots n_{f}$. Because of the feedback introduced, the recurrent neural model may be realized by a three-layer MLP.

This is illustrated by the example given in Fig. 5 which shows a residual evaluation scheme processing three residuals $\left(r_{1}, r_{2}, r_{3}\right)$ to diagnose three faults $\left(f_{1}\right.$, $\left.f_{2}, f_{3}\right)$.

The corresponding neural network has the following architecture: an input layer with 12 units representing all possible states of the fuzzy residuals together with the past decisions, a hidden layer having 4 units, and an output layer with 3 units each assigned to a decision function. The use of this RNN architecture ensures reliable dynamic decision making (Alexandru et al. 2000; Benloucif and Mehennaoui, 2005 and Chen and Lee 2002).

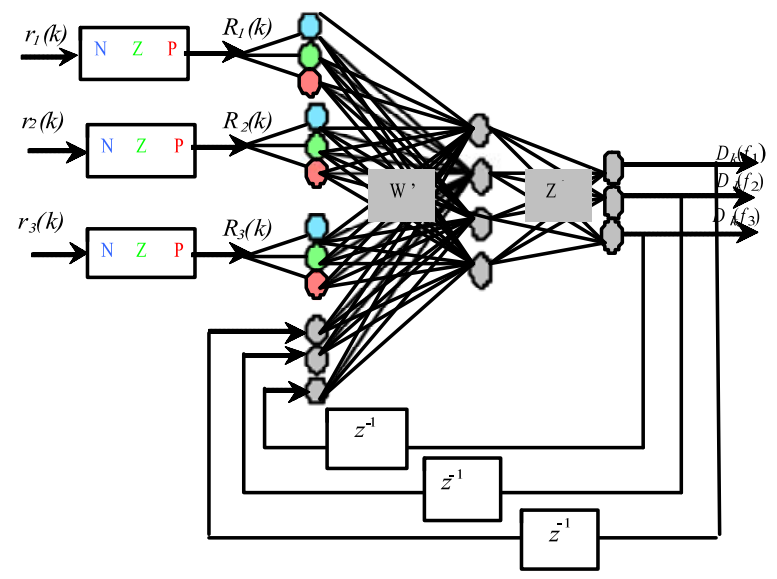

Figure 5. Example of a RNN used for residual evaluation

\subsubsection{Training}

Prior to on-line use, network training is performed for all possible fault scenarios. During training a residual pattern corresponding, eg. to fault $f_{l}$, is applied to the network input and a one is assigned to the corresponding output. The network weights are then adjusted by an appropriate algorithm thus enabling the neural network to learn the imposed input-output pattern. The use of the backpropagation algorithm is recommended (Benloucif and Mehennaoui 2005). The ultimate goal of the training is to achieve the extraction and selection of the necessary parameters defining the «If-Then» inference rules

\section{Numerical Results}

Results using MATLAB simulation are next presented to assess the ability of this diagnosis approach based on neural and fuzzy techniques to detect and isolate sensor faults in an induction motor. Its model expressed in the two-phase reference frame $(d, q)$ is given by the nonlinear state space Eq. (5).

The squirrel-cage induction motor considered here has power rating of $1 \mathrm{~kW}$ and its electrical and mechanical parameters are as follows:

$$
\begin{aligned}
& R_{s}=10(\Omega), R_{r}=5(\Omega), L_{s}=500(\mathrm{mH}), \\
& L_{r}=430(\mathrm{mH}) L_{m}=430(\mathrm{mH}), J=0.08\left(\mathrm{~kg} . \mathrm{m}^{2}\right), \\
& T_{L}=1 \quad(\mathrm{~N} . \mathrm{m}), f_{v}=0.025(\mathrm{~N} . \mathrm{m} . \mathrm{s} / \mathrm{rad}), p=2 .
\end{aligned}
$$

Simulation is carried out with a sampling period of $1 \mathrm{msec}$, with $400 \mathrm{~V}$ and $50 \mathrm{~Hz}$ sinusoidal inputs. In normal operation, the outputs $\left(I_{s d}, I_{s q}, \Omega\right)$ and the electromagnetic torque $T_{e}$ are shown in Fig. 6. 

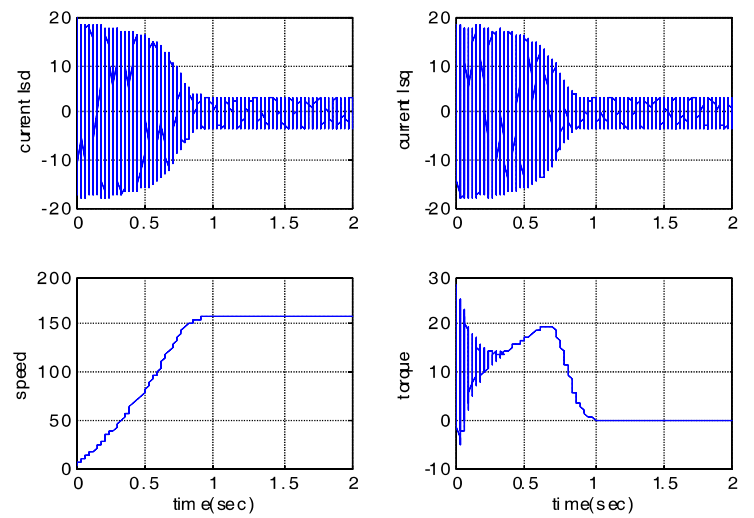

Figure 6. Stator currents $\left(I_{s d}, I_{s q}\right)$, rotor speed $\Omega$, torque $T_{e}$ (normal operation)

\subsection{Residual Generation:}

A NNARX model having the architecture shown in Fig. 3 has been used with the following parameters: $n_{1}$ $=n_{2}=n_{3}=n_{1}=m_{1}=m_{2}=1, d=1$. Training of this MLP network was achieved by the LevenbergMarquardt algorithm for different numbers of hidden neurons. For $n_{h}=4$, the output error cost reached at 36 iterations is $E=1.528 \mathrm{e}-002$. After validation this NNARX model is used to generate the residuals:

$$
r_{i}(k)=y_{i}(k)-\hat{y}_{i}(k), i=1 \ldots 3
$$

\subsection{Residual Evaluation}

The linguistic variables describing the fuzzified residuals are defined by the following membership functions (MF): «N: negative residual with trapezoidal $\mathrm{MF}\rangle,\langle Z$ : zero residual with triangular $\mathrm{MF} »,\langle\mathrm{P}$ : positive residual with trapezoidal MF ».

After many tests on residuals for different fault sensor situations to achieve a good trade off between missed detections and false alarms, the following membership functions for each residual were selected:

Residual 1: N1 $=[-1,-1,-0.005,-0.002]$

$\mathrm{Z} 1=[-0.0025,0,0.0045], \quad \mathrm{P} 1=[0.0035,0.006,1,1]$.

Residual 2: N2 $=[-1,-1,-0.04,-0.015]$

$\mathrm{Z} 2=[-0.02,0,0.005], \mathrm{P} 2=[0.004,0.009,1,1]$.

Residual 3: N3 $=[-1,-1,-0.018,-0.015]$

$\mathrm{Z} 3=[-0.016,-0.0135,-0.012], \mathrm{P} 3=[-0.0125,-0.0115,1,1]$.

The RNN used in this simulation study is shown in Fig. 5. Its training is based on the rules summarized in Table 1 which have been obtained after many simulation tests. The learning operation realized by the backpropagation algorithm converged after 3600 epochs with a sum of squared error $\mathrm{E}=0.025$.

Each row of the Inference table represents a rule. For example, rule 2 is expressed as:

IF \{residual 1 is positive and residual 2 is negative and residual 3 is zero THEN sensor 1 is faulty.
Table 1. Inference table

\begin{tabular}{|l|l|l|l|l|l|l|l|l|l|l|l|l|}
\hline $\mathrm{N}^{\circ}$ & $\mathrm{N} 1$ & $\mathrm{Z} 1$ & $\mathrm{P} 1$ & $\mathrm{~N} 2$ & $\mathrm{Z} 2$ & $\mathrm{P} 2$ & $\mathrm{~N} 3$ & $\mathrm{Z} 3$ & $\mathrm{P} 3$ & $\mathrm{D} 1$ & $\mathrm{D} 2$ & $\mathrm{D} 3$ \\
\hline 1 & 0 & 1 & 0 & 0 & 1 & 0 & 0 & 1 & 0 & 0 & 0 & 0 \\
\hline 2 & 0 & 0 & 1 & 1 & 0 & 0 & 0 & 1 & 0 & 1 & 0 & 0 \\
\hline 3 & 0 & 0 & 1 & 0 & 0 & 1 & 0 & 1 & 0 & 0 & 1 & 0 \\
\hline 4 & 0 & 1 & 0 & 0 & 1 & 0 & 0 & 0 & 1 & 0 & 0 & 1 \\
\hline 5 & 0 & 0 & 1 & 1 & 0 & 0 & 0 & 0 & 1 & 1 & 0 & 1 \\
\hline 6 & 0 & 0 & 1 & 0 & 0 & 1 & 0 & 0 & 1 & 0 & 1 & 1 \\
\hline 7 & 0 & 0 & 1 & 0 & 0 & 1 & 0 & 1 & 0 & 1 & 1 & 0 \\
\hline
\end{tabular}

\subsection{Sensor Fault Diagnosis of the Induction} Motor

Various simulation tests have been performed in order to validate the efficiency of this diagnosis scheme and the results are quite conclusive. Bias and drift type sensor faults are introduced during steady state conditions of the system. For illustrative purposes only a few fault scenarios summarized in Tables 2 to 4 are discussed.

Table 2. Case 1

\begin{tabular}{|c|c|c|}
\hline Sensor $\mathrm{N}^{\circ}$ & Fault time & Bias fault \\
\hline 1 & 2.5 & 0.2 \\
\hline
\end{tabular}

Table 3. Case 2

\begin{tabular}{|c|c|c|}
\hline Sensor $\mathrm{N}^{\circ}$ & Fault time & Bias fault \\
\hline 2 & 2 & 0.8 \\
\hline 3 & 3 & 3 \\
\hline
\end{tabular}

Table 4. Case 3

\begin{tabular}{|c|c|c|}
\hline Sensor $\mathrm{N}^{\circ}$ & Fault time & Drift slope \\
\hline 2 & 2 & 0.001 \\
\hline 3 & 3 & 0.0025 \\
\hline
\end{tabular}

\subsubsection{Case 1}

A bias type fault is injected on sensor 1 as described in Table 2.

The corresponding residuals are shown in Fig. 7. Although a single fault may induce changes in several residuals ( here a fault on sensor 1 affects positively the first residual and negatively the second residual at time $\mathrm{t}=2.5 \mathrm{sec}$ ) the decision functions ensure successful detection and isolation of the fault on sensor 1 as shown in Fig. 7. The neuro-fuzzy classifier has been trained to recognize the faulty situations from the fuzzified residual patterns according to the rule base given in Table 1.

\subsubsection{Case 2}

This fault scenario of bias faults on sensors 2 and 3 is described in Table 3.

The residuals and the corresponding decision functions are shown in Fig. 8. The faulty sensors are promptly detected and correctly isolated.

\subsubsection{Case 3}

This fault scenario uses drift faults on sensors 2 and 
3 as described in Table 4. Drift faults are modelled as ramp functions with given slopes.

The diagnosis effectiveness in the presence of sensor drift faults is illustrated in Fig. 9. We notice a detection delay for fault sensor 2. This delay, which is dependent on the slope of the drift, gives rise to a temnorarv false alarm on sensor 1 .
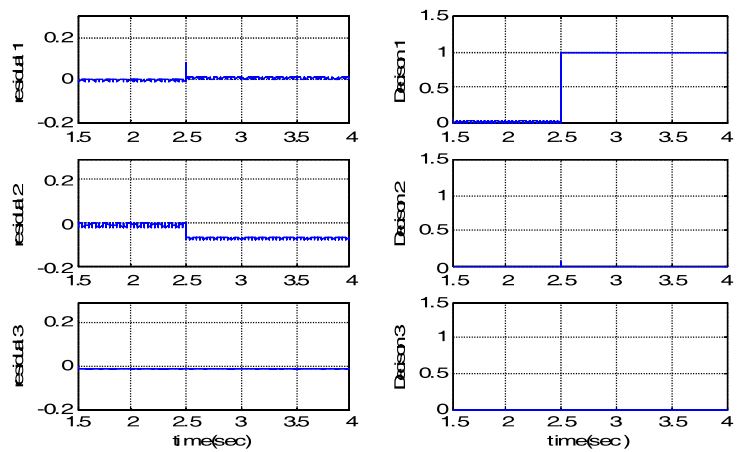

Figure 7. Faculty residuals and corresponding decisions (Case 1)
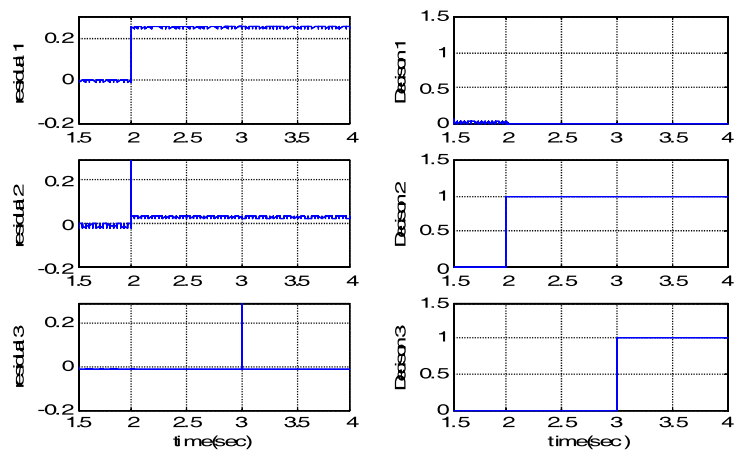

Figure 8. Faculty and corresponding decisions (Case 2)
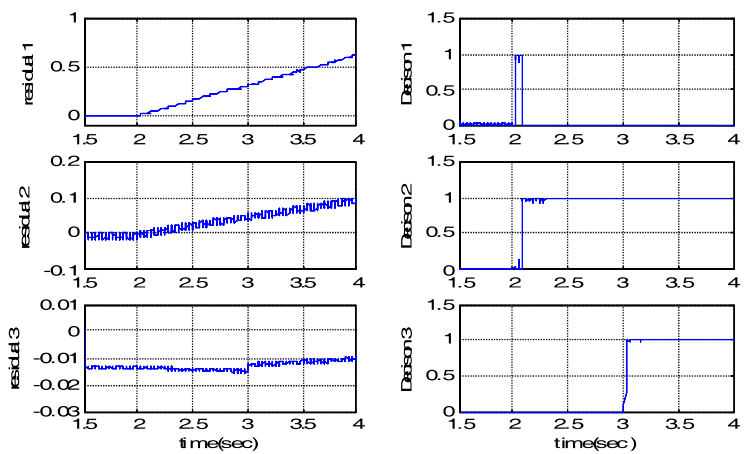

Figure 9. Faculty residuals and corresponding decisions (Case 3)

\section{Conclusions}

In this paper, a neuro-fuzzy scheme for on-line fault diagnosis was applied to the induction machine. This FDI approach relies on combinations of neural modelling and fuzzy logic which can deal effectively with nonlinear dynamics and uncertainties.

The proposed neuro-fuzzy FDI scheme is based on a two step procedure: a neural NNARX model is used for residual generation and a recurrent fuzzy neural network performs the residual evaluation task. Fault diagnosis is achieved by training the network to recognize the fault signatures from the patterns of the fuzzified residuals. The successful results obtained in simulation demonstrate the efficiency of this neuro-fuzzy diagnosis scheme to detect and isolate bias and drift sensor faults in an induction motor.

\section{References}

Alexandru, M., Combastel, C. and Gentil, S., 2000, "Diagnostic Decision using Recurrent Neural Networks," Proc. IFAC Safeprocess 2000, Budapest, Hungary.

Babuska, R., 1998, "Fuzzy Modeling for Control," Kluwer Academic Publishers.

Benloucif, M.L. and Staroswiecki, M., 2002, "Fault Diagnosis using a Robust Estimation Method," Proc. International Conference CIFA-2002, Nantes, France.

Benloucif, M.L. and Mehennaoui, L., 2002, "A Mixed Analytical Neuro-Fuzzy Approach for Fault Diagnosis," Proc. 2ND Instrumentation and Measurement in Petroleum Applications Conference IMPAC-2002, Boumerdès, Algeria.

Benloucif, M.L. and Mehennaoui, L., 2005, "A Fuzzy Neural Scheme for Fault Diagnosis," Proc. International Computer Systems and Information Technology Conference ICSIT'05, Algiers, Algeria.

Benloucif, M.L. and Balaska, H., 2006, "Robust Fault Detection for an Induction Machine," 7th World Automation Congress- WAC 2006, Budapest, Hungary.

Then, Y.M. and Lee, M.L., 2002, "Neural Networks Based Scheme for System Failure Detection and Diagnosis," Mathematics and Computers in Simulation, 58, pp. 101-109.

Evsukoff, A., Combastel, C. and Gentil, S., 1999, "Qualitative Reasoning and Neural Network Decision Procedures for Fault Detection and Isolation," Proc. 14th IFAC World Congress, Beijing, China.

Frank, P.M., 1990, "Fault Diagnosis in Dynamic Systems using Analytical and Knowledge Based Redundancy - A Survey and Some New Results," Automatica, 26, pp. 459-474.

Frank, P.M., 1994, "Application of Fuzzy Logic to Process Supervision and Fault Diagnosis," Proc. IFAC Safeprocess 94, Espoo, Finland.

Garcia, E. and Frank, P.M., 1997, "Deterministic Nonlinear Observer Based Approaches to Fault Diagnosis: A survey," Control Eng. Practice, vol. 5(5), pp. 663-670. 
Isermann, R., 1998, "On Fuzzy Logic Applications for Automatic Control, Supervision and Fault Diagnosis," IEEE Trans. on Systems, Man and Cybernetics, Vol. 28(2).

Jiang, B., Staroswiecki, M. and Cocquempot, V., 2001, "Robust Observer Based Fault Diagnosis for a Class of Nonlinear Systems with Uncertainty," Proc. 40th IEEE Conference on Decision and Control, CDC'01, Orlando, USA.

Mamdani, E. and Assilian, S., 1995, "An Experiment in linguistic Synthesis with Fuzy Logic Controller," Int. J. Man-Machine Studies, 7(1).

Norgaard, M., Ravn, O., Poulsen, N.K.. and Hansen, L.K., 2000, "Neural Network for Modelling and Control of Dynamic Systems," Springer Verlag, London.

Patton, R. and Chen, J., 1997, "Observer-Based Fault Detection and Isolation: Robustness and Applications," Control Eng. Practice, Vol. 5(5), pp. 671-682.

Schneider, H. and Frank, P.M., 1996, "Observer Based
Supervision and Fault Detection in Robots using Nonlinear and Fuzzy Logic Residual Evaluation," IEEE Trans. on Control System Technology, Vol. 4(3), pp. 274-282.

Simani, S. and Fantuzzi, C., 2002, "Neural Networks for Fault Diagnosis and Identification of Industrial Processes," Proc. of the 10th European Symposium on Artificial Neural Networks, Bruges, Belgium.

Takagi, T. and Sugeno, M., 1985 "Fuzzy Identification of Systems and Applications to Modelling and Control," IEEE Trans. On system, Man and Cybernetics, Vol. 15(1).

Theilliol, D., Sauter, D. and Vela Valdes, L.G., 1997, "Integration of Qualitative and Quantitative Methods for Fault Detection and Isolation," Proc. IFAC Safeprocess 97, Hull, U.K.

Uppal, F.J., Patton, R.J. and Palade, V., 2002, "NeuroFuzzy Based Fault Diagnosis Applied to an Electro- Pneumatic Valve," 15th IFAC World Congress, Barcelona, Spain. 